\title{
Single Stages Repair of Hypospadias Using The Snodgrass Technique: A Modern Choice
}

\author{
Karn $\mathrm{B}^{1}$, Shrestha NM ${ }^{2}$
}

\begin{abstract}
Background: Proximal hypospadias with chordee is the most challenging variant of hypospadias to reconstruct. During the last 10 years, the approach to severe hypospadias has been controversial. Materials \& Methods: This is a hospital based observational study conducted in the plastic surgery unit of department of surgery at Nepalgunj Medical College and Teaching Hospital Kohalpur from 2012 March to 2016 September. All patients underwent single staged procedures using Snodgrass technique. Results: Thirty three patients were operated. Twenty four patients had no complications. Single fistula was present in 6(18.18\%) patients. Two (6.06\%) patients had 2 fistulae; one at the original site of the hypospadias and the other was sub-coronal. One (3.03\%) patient had moderate meatal stenosis, which was successfully treated by repeated dilatations. Conclusion: Single Staged procedure using the principles of Snodgrass's technique; is a versatile operation that can be used for the proximal hypospadias. It decreases the rate of fistula formation; disruption; stenosis and gives a satisfactory cosmetic appearance.
\end{abstract}

Key words: proximal hypospadias, single staged repair

\section{INTRODUCTION}

Hypospadias is the third most common congenital deformity after clubfoot andhydrocele, with an incidence of 3-4 per 1000 live births. Rarely, it may be accompanied by undescended testis, kidney anomalies, hernias and other congenital anomalies. There have been many operations described for repair of hypospadias, but non of the methods have been proven ${ }^{1}$.

Proximal hypospadias with chordae is the most challenging variant of hypospadias to reconstruct ${ }^{2}$. During the last 10 years, the approach to sever hypospadias has been controversial ${ }^{3}$. This controversy exists with regard to the best approach to proximal hypospadias. Although 1-stage repair have been shown to be successful for some forms of proximal hypospadias, many still favor a more traditional staged approach when sever chordae is present to achieve adequate straightening and lengthening of the penis at the time of the first stage repair. This is achieved either by division of the urethral plate or Byars's flaps are created and mobilized ventrally to cover the ventral shaft of the penis ${ }^{4}$.

\section{MATERIALS AND METHODS}

This is a hospital based observational study conducted in the plastic surgery unit of department of surgery at Nepalgunj Medical College and Teaching Hospital Kohalpur from 2012 March to 2016 September.

All the patients were admitted. Their age, history and

1. Dr. Binod Karn

2. Dr. N. M. Shrestha

\section{Address for correspondence}

Dr. Binod Karn

Department of Surgery, Burn \& Plastic Unit

Nepalgunj Medical College \&Teaching Hospital

Kohalpur, Banke, Nepal

Email:drbin2007@gmail.com examination findings were recorded. The type of hypospadias and association with chordee were recorded. Patients were followed up till 1 year.

All surgeries were performed by the same surgeon. All surgeries were performed under general anaesthesia. The patients were at least 2 years to have acceptable size structures. A 4 /0 Polypropylene stitch was placed on the glans for traction and a suitable urethral catheter was inserted. A circumferential dorsal incision was made about half $\mathrm{cm}$ from the base of the glans. This was advanced ventrally till $2 \mathrm{~mm}$ from the urethral plate; then proximally along the plate till it passes along the proximal edge of the urethral meatus. Complete degloving of the penile shaft wasperformedtill its base. Then artificial erection was performed to demonstrate any residual chordee. If this persisted; transection of the urethral plate is done just proximal to the glans.

Urethral plate was tubularized around the catheter to make the new urethra using 6/0 Polyglactin continuous suture. Appropriate width of the tube was confirmed to allow the catheter to be removed without tearing the neo-urethra. A second layer from the penile adventitial tissues was raised and sutured over the neo-urethra making sure that the two suture lines are not directly over each other and the second layer was mobilized enough to reach the glans tip. At last, the penile skin was sutured as a third layer. The dressing was removed after 48 hours and the catheter after 7-10 days.

\section{RESULTS}

Thirty three patients were operated. The age ranged from $1-22$ (Table I). Sixteen patients had proximal penile hypospadias, 17 had penoscrotal hypospadias(Table II). In 17 patients with penoscrotal hypospadias the chordee present.

Twenty four patients had no complications. Single fistula was present in $6(18.18 \%)$ patients at the original site of the hypospadias and was re-closed after 6-9 months. Two patients had $2(6.06 \%)$ fistulae. One at the original site of the 
hypospadias and the other was sub coronal. They were closed after one year of recurrence. There were no recurrence in reoperated cases. One $(3.03 \%)$ patient had moderate meatal stenosis, which was successfully treated by repeated dilatations. There was no recurrence of chordee. None of the patients needed neither skin nor bladder mucosal grafts. The complete disruption and the urethral diverticula didn't occur in any of the patients (Table III).

\begin{tabular}{|c|c|c|}
\hline Age group (years) & Number of cases & Percentage \\
\hline $1-5$ & 9 & 27.27 \\
\hline $6-10$ & 13 & 39.39 \\
\hline $11-15$ & 7 & 21.21 \\
\hline 16 and above & 4 & 12.12 \\
\hline
\end{tabular}

Table I : Age distribution of cases by age

\begin{tabular}{|c|c|}
\hline Type of hypospadias & No. of patients \\
\hline Proximalpenile Hypospadia & $16 \%$ \\
\hline Penoscrotal hypospadias & $17 \%$ \\
\hline
\end{tabular}

Table II: Types of hypospadias

\begin{tabular}{|c|c|}
\hline Complication & No. of patients \\
\hline No complications & 24 \\
\hline Single fistula & 6 \\
\hline Double fistulae & 2 \\
\hline Meatal stenosis & 1 \\
\hline Urethral diverticula & 0 \\
\hline Recurrence of chordae & 0 \\
\hline Total & 33 \\
\hline
\end{tabular}

Table III: incidence of complications
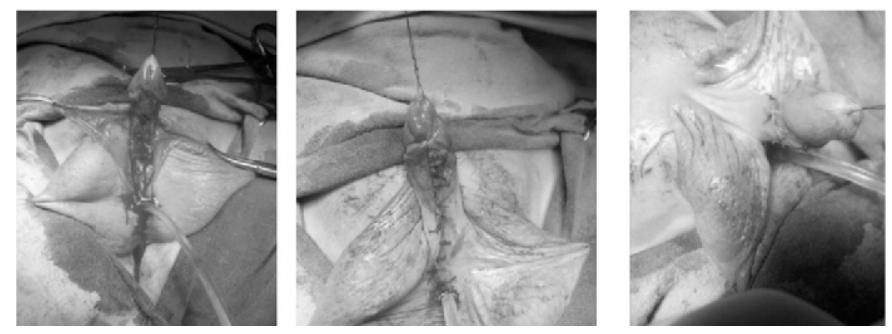

Figure1: Intra operative
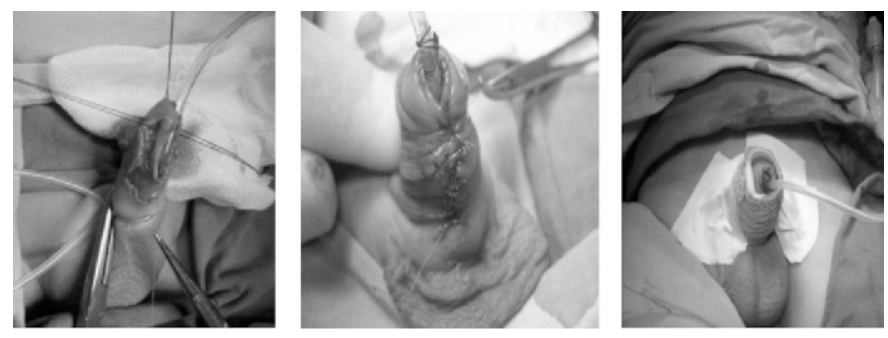

Figure 2: Post operative

\section{DISCUSSION}

Surgery for hypospadias, especially the more proximal types, has always been a tricky and difficult operation. This is shown by the number of operations described in the literature on the surgical treatment of this congenital deformity. Over the years, many techniques have evolved ranging from one stage procedure, where correction of chordee and simultaneously creation of the new urethra, to two stages procedure; where creation of the new urethra is done in a separate operation ${ }^{5}$.

Horton et al reported that only $10 \%$ of all hypospadias cases are in the proximal shaft of the penis, penoscrotal or perineal ${ }^{6}$. This group of hypospadias patients represent a major challenge to the paediatric urologist, with a higher incidence of recurrence and complication than the more proximal cases.

In this series, Pyar's 2 staged operation was used in all 33 cases with proximal hypospadias. This operation is very versatile because it can be used to treat any type of hypospadias whether proximal or distal. Being a vascularized skin flap, it is very reliable when used in the first stage, providing a vascularized skin available to resurface the raw area that has been created as a result of chordee correction. This need for a large vascularized flap to cover this raw area is especially needed after release of chordee in the more proximal hypospadias. The other advantage of Pyar's operation is that on completion of the procedure, there is no need to do circumcision ${ }^{7}$ which is a big advantage to our patients.

The size of the neo-urethra is of utmost importance; it must be adequate enough to prevent tearing of the urethra when the catheter is removed. I think if we put a very small catheter (4 or $5 \mathrm{Fr}$ ) at the end of the second stage operation, we may abolish the need for a supra-pubic catheter; making the wound dry if cautiously kept patent all the time ${ }^{5}$. Tears in an already fragile tissue often lead to fistula formation. The second layer is also important in providing an extra layer to prevent fistula formation.

Complication of surgery for hypospadias are complete disruption, fistula formation, stenosis, post operative bleeding, , recurrence of chordee or excess skin at the glans. The most common complication is fistula formation . Various factors have been implicated in fistula formation such as infection, trauma and poor healing. Other factors may include back pressure, turbulence, narrowing, skin bridges or crests on irregular surface of the new urethra. The incidence of fistula formation is reported to be from $3 \%$ to $50 \%$ and it is higher in the more proximal hypospadias ${ }^{8}$. In this series, the fistula rate was $24.2 \%$ ( 8 out of 33 patients). These fistulae were small and easily closed without any further complication.

A single case with meatal stenosis was successfully managed by repeated dilatation without general anesthesia. This low rate of meatal stenosis could be attributed to the size of the neo- 
urethra which was made wide enough. There was no complete disruption in any of the cases in this series nor recurrence of chordee. Satisfactory cosmetic results were achieved in all cases.

\section{CONCLUSIONS}

Single staged procedure using the principles of Snodgrass's technique is a versatile operation that can be used for the all types of hypospadias. It decreases the rate of fistula formation, disruption and stenosis, and give a satisfactory cosmetic appearance.

\section{REFERENCES}

1. Arshad AR. Hypospadias repair : Pyar's two stage operation revisited. Br J Plast Surg 2004; 58: 481-86.

2. Defoor W, Wacksman J. Results of single staged hypospadias surgery to repair penoscrotal hypospadias with bifid scrotum or penoscrotal transposition. J Urol 170: 1585-1588, 2003.

3. Shapiro SR. Fistula repair. Reconstructive and plastic surgery of the external genitalia: adult and paediatric; London: WB Saunders; 132-6; 1999.

4. Bracha A.. Hypospadias repair : the two stage alternative . $\mathrm{Br} \mathrm{J}$ Urol 76 (suppl) :31-41,1995.

5. Aseem RS, Rakesh PP, Douglas AC. The 2-stage hypospadias repair ; is it a misnomer. J Urol 172: 1714-1716, 2004.

6. Horton CE, Sadove Rc, Devine CJ. Reconstruction of the male genital defects: congenital. In: McCarthy JG, editor. In: Plastic surgery, Vol 6, 4153-79, 1990.

7. Earl Y.C. , Bradley P.K., John C.P. et al . Proximal division of the urethral plate in staged hypospadias repair. J Urol 170: 1580$1584,2003$.

8. Byars LT. A technique for consistently satisfactory repair of hypospadias. Surg Gynecol Obstet 100: 184-90; 1955. 